\title{
Temporal artery biopsies in south-east Scotland: a five-year review
}

S Gajree ${ }^{1}$, S Borooah ${ }^{2}$, N Dhillon ${ }^{3}$, C Goudie $^{4}$, C Smith $^{5}$, P Aspinall ${ }^{6}$, B Dhillon ${ }^{7}$

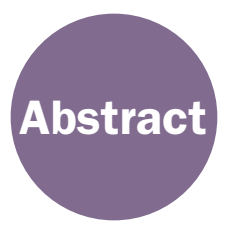

Temporal artery biopsy is the gold standard investigation for the diagnosis of giant cell arteritis. The aim of this retrospective study was to investigate the use of temporal artery biopsy in diagnosing giant cell arteritis in southeast Scotland over a five-year period. We aimed to quantify success rates, and predictive factors for a positive biopsy, as well as compare the different specialities performing the biopsies. The data should enable the development of better criteria for referral for investigation of giant cell arteritis.

Methods Patients were identified using a database of temporal artery biopsies generated by the pathology department in NHS Lothian (south east Scotland), for all biopsies examined between January 2010 and December 2015. An electronic patient record was used to retrospectively examine the records of patients in the database.

Results A total of 715 biopsies were included in the study, of which 250 (35.0\%) showed features of giant cell arteritis. The main predictors for a positive biopsy were age at biopsy, specialty performing biopsy, erythrocyte sedimentation rate, jaw claudication/pain, and ophthalmic symptoms. The most important predictor of a positive biopsy was erythrocyte sedimentation rate. The length of biopsy was not found to be a predictor of positive biopsy; however, diameter of biopsy was predictive.

Conclusions We have shown that many temporal artery biopsies are negative, and finding ways to reduce the number of patients unnecessarily undergoing biopsy will be essential in reducing workload and streamlining services. This study demonstrates some key predictive factors for patients with positive biopsies. The study also shows that a large proportion of biopsies taking place do not result in the recommended length of specimen, but this does not necessarily reduce the likelihood of a positive biopsy.

Keywords: giant cell arteritis, temporal arteritis, temporal artery biopsy

Declaration of interests: No conflict of interests declared

\section{Introduction}

Giant cell arteritis (GCA) is a large vessel vasculitis. ${ }^{1}$ It is the most common systemic vasculitis, and is characterised by a granulomatous inflammation of larger arteries, most frequently affecting the temporal, ophthalmic, posterior ciliary and vertebral arteries. ${ }^{2}$ GCA has a varied clinical presentation. Classical symptoms include headache, jaw claudication, fatigue, loss of appetite and scalp tenderness. However, the most devastating complication is an acute irreversible loss of vision resulting from arteritic occlusion of the ophthalmic artery, which can lead to complete sight loss. Visual loss occurs in up to $20 \%$ of patients and can be an early manifestation of the disease but is preventable with the timely administration of systemic corticosteroids..$^{3,4}$
Correspondence to:

$S$ Gajree

3/2, 267 Crow Road

Glasgow G11 7BE

UK

Email:

sonulgajree@gmail.com
Although GCA is diagnosed clinically, supported by a derangement of blood markers, ${ }^{5}$ a definitive diagnosis can be difficult given the non-specific nature of symptoms. GCA typically affects patients over the age of 50 , and the incidence continues to increase with an ageing population. ${ }^{6}$ To prevent acute vision loss, steroid therapy should be initiated when there is clinical suspicion, but urgent accurate diagnosis of GCA is required for long-term management.

Temporal artery biopsy (TAB) is the gold standard investigation for the confirmation of GCA. Early TAB is recommended in all patients with suspected temporal arteritis due to its diagnostic and prognostic role. ${ }^{6}$ Positive TABs demonstrate disruption of the internal elastic lamina with a mononuclear 
Figure 1 Haematoxylin and eosin stained section from a TAB demonstrating active temporal arteritis, with disruption of the internal elastic lamina with a mononuclear cell infiltrate. The arrow points to the narrowed lumen, while triangular arrowheads demonstrate multinucleate giant cells bordering the elastic lamina

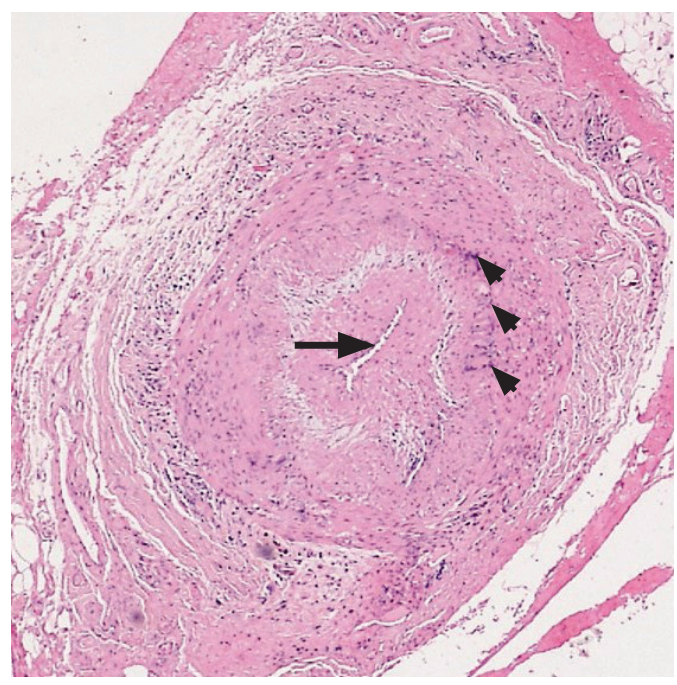

cell infiltrate (Figure 1). ${ }^{7}$ However, it has been suggested that TABs are associated with a high false negative rate due to the occurrence of skip lesions and are not without risk, including facial nerve injury, pain, surgical wound complications and stroke. ${ }^{8}$

In the UK, a range of specialties perform TABs including neurosurgery, ophthalmology, vascular surgery and general surgery teams. The broad range of symptoms also mean that a number of different specialities refer patients for TAB. TABs are ideally performed within two weeks of the commencement of systemic corticosteroid treatment to attain the best yield, as steroid therapy may result in complete suppression of the inflammatory response..$^{9,10}$ This leads to a large number of acute referrals, placing increasing strain on services.

The aim of this retrospective study was to investigate the use of TAB in diagnosing temporal arteritis in south-east Scotland over a five-year period. We aimed to quantify success rates, and predictive factors for a positive biopsy, as well as compare the different specialities performing the biopsies. The data should enable the development of better criteria for the referral and investigation of GCA.

\section{Methods}

Patients were identified using a database of TABs generated by the pathology department in NHS Lothian, Edinburgh, for all biopsies examined between January 2010 and December 2015. An electronic patient information record was used to retrospectively examine the records of patients in the database. Baseline demographic data was collected as well as data regarding presenting symptoms and initial erythrocyte sedimentation rate (ESR) and C-reactive protein(CRP). The speciality performing the biopsy was recorded and pathology reports were assessed for biopsy length, diameter, and histological result. Pathological assessment was based on haematoxylin and eosin stained sections, with elastic Van
Figure 2 Histological results of temporal arterial biopsy, speciality from which patients were referred for biopsy, and speciality performing biopsy

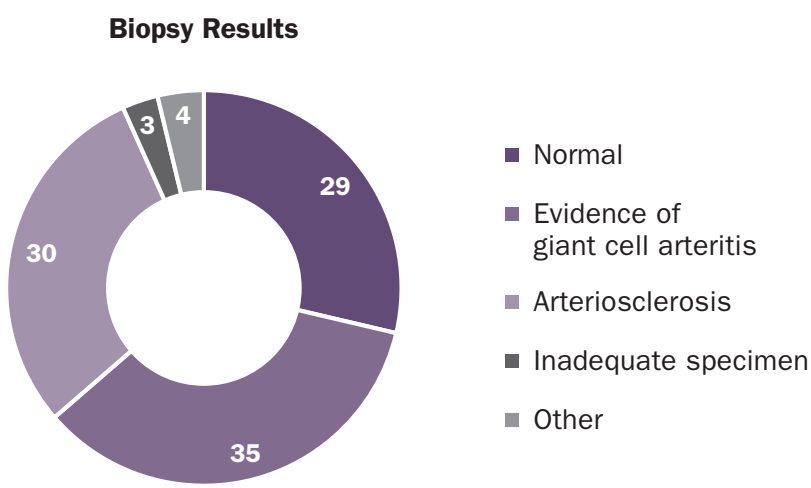

Referral Speciality
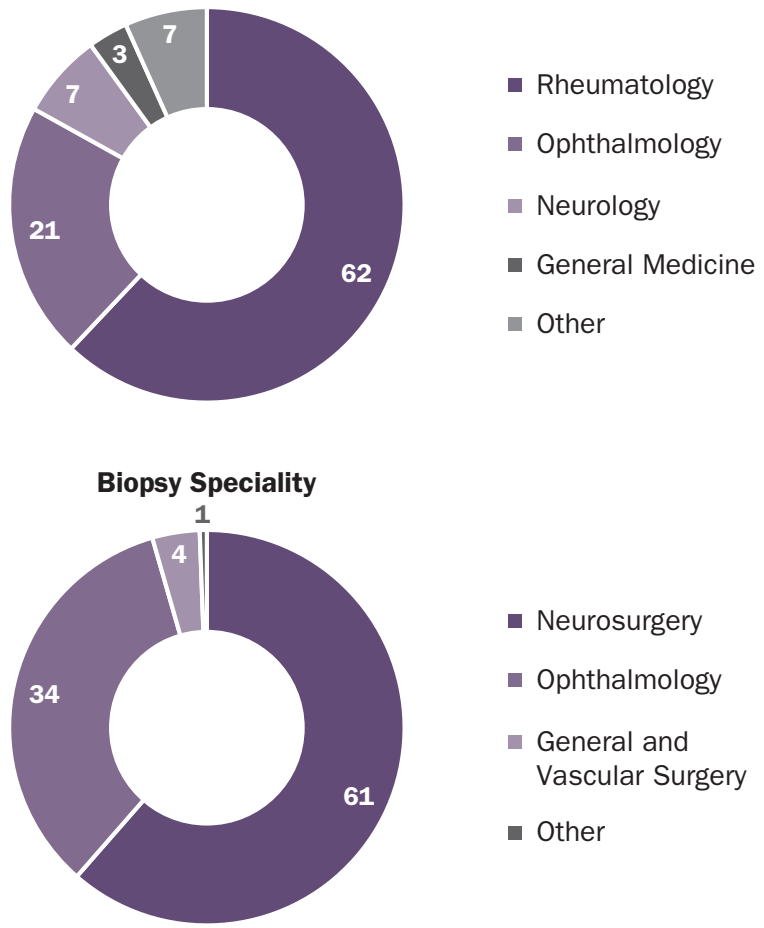

Gieson stained sections to assess internal elastica damage. Peri-operative corticosteroid dosing was also noted.

SPSS (IBM Corp, Armonk, NY) statistics software was used to collate and perform statistical analysis on the data collected, and a $p$ value of less than 0.05 was deemed significant. A correlated component logistic regression was run with $\mathrm{M}$ fold cross-validation to find variables that predicted a positive histopathological diagnosis of GCA (CORExpress, Statistical Innovations Inc.). See Appendix 1 for details.

\section{Results}

In total, 715 TABs were included in our study. These were all performed in the south-east of Scotland, from 2010 to 
2015. Of these biopsies, 496 were female (69.4\%) and 219 (30.6\%) were male, with an overall mean age of 71 (SD 10.4) at time of biopsy. Of the 715 biopsies included in the study, 250 (35.0\%) biopsies showed features of GCA, 205 (28.6\%) were completely normal, 212 (29.7\%) showed signs of arteriosclerosis and 21 (2.9\%) were deemed inadequate for diagnosis (Figure 2).

The mean age of those patients with a positive biopsy was 73.8 (SD 9.4), compared to a mean age of 69.8 (SD 10.1) in those with a negative biopsy (unpaired t test, $p<0.0001$ ). There was no statistical association between gender and histological diagnosis of temporal arteritis (two tailed z-score test, $p=0.28$ ). Two patients with positive biopsies were under 50 years (ages 40 and 47). The oldest positive biopsy was from a patient aged 94.

The most common symptom reported was headache, with $440(61.5 \%)$ referrals mentioning headache at presentation. Other common symptoms included visual disturbance or other eye symptoms $(219,30.6 \%)$, scalp tenderness (206, $28.8 \%)$, and jaw claudication (124, 17.5\%).

A number of different specialities referred patients for TABs. We were able to establish the referring speciality in 490 biopsies. Of these, 304 (62.0\%) came from rheumatology, 103 (26.4\%) from ophthalmology, and 34 (6.9\%) from neurology. The remaining referrals came from a variety of specialities, including general medicine, infectious diseases, medicine of the elderly, and emergency medicine.

Of the 304 patients who were referred for TAB by rheumatologists, 94 (30.9\%) showed features of temporal arteritis, while 49 (47.5\%) of the 103 ophthalmology referrals were positive for temporal arteritis (two tailed z-score test, $p=0.002)$.

The TABs were performed by several different specialities, including ophthalmology, neurosurgery, general and vascular surgery. A total of 199 (27.8\%) of the biopsy pathology reports did not include details of performing speciality. Of the remaining 516 biopsies, 317 (61.4\%) were performed by neurosurgeons, 176 (34.1\%) by ophthalmologists, and 20 (3.9\%) by general and vascular surgeons.

Overall mean biopsy length was $11.64 \mathrm{~mm}$ (SD 6.4), with a median of $10 \mathrm{~mm}$ and interquartile range of $8-15 \mathrm{~mm}$. The mean length of the 317 biopsies performed by neurosurgery was $12.22 \mathrm{~mm}$ (SD 6.97), and the mean length of the 176 biopsies performed by ophthalmology was $11.7 \mathrm{~mm}$ (SD 4.9) (unpaired test, $p=0.39$ ). The overall mean diameter of biopsy was $2.35 \mathrm{~mm}$ (SD 1.05)

Four hundred and twenty biopsies were $10 \mathrm{~mm}$ or longer. Of these, 157 (37.4\%) showed evidence of temporal arteritis. Two hundred and forty-one biopsies were less than $10 \mathrm{~mm}$ in length, with 80 (33.2\%) showing evidence of temporal arteritis (two tailed $z$-score test, $p=0.28$ ). Ninety-four biopsies measured $20 \mathrm{~mm}$ or greater, with 38 (40.4\%) of these demonstrating evidence of GCA (two tailed z-score test compared with biopsies less than $10 \mathrm{~mm}, \mathrm{p}=0.21$ ).

We were able to establish the ESR prior to biopsy in 546 patients. Of these, the mean ESR was 57 (SD 30.3). Of the 250 patients with biopsies showing signs of temporal arteritis, we established the ESR prior to biopsy in 204 cases, with a mean ESR of 65 (SD 29.7). From those with a negative biopsy, pre biopsy ESR was established in 340 cases, with a mean ESR of 52 (SD 29.8), (unpaired t test, $p<0.0001$ ).

CRP was recorded prior to biopsy in 188 patients. In those with positive biopsies, the mean CRP was 76 (SD 69.8), while in those with negative biopsies the CRP was 61 (SD 76.8) (unpaired test, $p=0.20$ ).

Body mass index (BMI) was recorded in 83 patients. There was a statistically lower mean BMI in patients with histological evidence of GCA (BMI 27.02, SD 4.7) than in patients with no evidence of GCA on biopsy (BMI 30.57, SD 7.2) (unpaired t test, $p=0.02$ ).

Output from our correlated component regression showed that the model was optimised with six predictors where $\mathrm{R}$ squared $=0.108$ and area under the curve $=0.687$. The main predictors for a positive biopsy were age at biopsy, specialty performing biopsy, ESR, jaw claudication/pain, and ophthalmic symptoms. Using Pratt's coefficient of importance, the most important predictor of a positive biopsy was found to be ESR (21\%) (see Appendix 1). The length of biopsy was not found to be a predictor of positive biopsy. However, diameter of biopsy was predictive.

\section{Discussion}

This study details the results of a large cohort of 715 patients who underwent TAB over a 5-year period in the southeast of Scotland. We aimed to quantify success rates and rates of biopsy by different speciality, but also to identify key predictors of positive biopsies in this population.

We have documented that referrals for TABs come from a wide variety of sources, with the majority coming from rheumatology and ophthalmology. There was a significantly larger proportion of referrals from ophthalmology which went on to show GCA on biopsy. This phenomenon has also previously been demonstrated by Moutray et al. ${ }^{11}$ This is likely due to the differences in clinical presentation of the patients presenting to the different specialities, with a larger proportion of patients with visual symptoms presenting to the ophthalmologists, and a larger proportion of patients with nonspecific generalised symptoms presenting to rheumatology. Moutray et al. also hypothesised that this difference may also stem from quicker access to biopsy for ophthalmologists compared with medical specialities.

It is well known that GCA is a disease which affects the elderly population, and previous studies have suggested a mean age of onset of $70 . .^{6}$ In our population, we saw an overall mean age at biopsy of 71 , but a significantly higher 
mean age in those with positive biopsies compared with those with negative biopsies ( 73.8 compared to 69.8 years). This echoes findings that the incidence of temporal arteritis increases with age. The American College of Rheumatology (ACR) classification criteria for diagnosing GCA includes age at disease onset being over $50 .{ }^{5}$ Although it is rare, two patients in our cohort had positive biopsies aged under 50 , with the youngest being just 40 . Despite the association with the elderly population, there have been several reports of GCA in younger age groups, ${ }^{11,12}$ including by Iraklis et al., who describe the case of a 17-year-old patient with biopsy proven GCA. ${ }^{13}$ It is therefore important not to overlook GCA as a possible diagnosis in younger patients presenting with other clinical features suggestive of GCA.

TABs are carried out by several different specialities in the south-east of Scotland. In our cohort of patients, neurosurgery and ophthalmology units performed the majority, with smaller numbers being performed by general and vascular surgeons. There was no statistically significant difference in the length of biopsies obtained by the neurosurgeons and the ophthalmologists, and the overall mean biopsy length was $11.64 \mathrm{~mm}$. Because of the nature of skip lesions in temporal arteritis, it is generally accepted that a longer biopsy is more useful at identifying the disease. ${ }^{12}$ The British Society of Rheumatology recommend that biopsy specimens should be no less than $1 \mathrm{~cm}$ and ideally greater than $2 \mathrm{~cm} .{ }^{6}$ Biopsies in south-east Scotland fell short of the $1 \mathrm{~cm}$ target in 241 (33.2\%) cases, and were over the ideal $2 \mathrm{~cm}$ in 94 (13.1\%) cases. These results are similar to those established by McMurran et al. in their recent case series detailing TABs in a district general hospital in the west of Scotland. ${ }^{13}$ There was no statistical difference, however, between the rates of positive biopsies among those biopsies over $2 \mathrm{~cm}$ and less than 1 $\mathrm{cm}$. This supports the findings of Mahr et al., who reviewed a dataset of 1,520 TABs, and demonstrated that there was no clear relationship between increasing $T A B$ length and GCA positivity. ${ }^{14}$ This may suggest that length of TAB is less important than previously thought.

A raised ESR is a cardinal finding in GCA, with some studies suggesting an elevated ESR is seen in $90-95 \%$ of patients with GCA. ${ }^{7}$ As such, an elevated ESR of above 50 is recognised in the ACR classification criteria for diagnosing GCA. ${ }^{5}$ The overall mean ESR seen in our cohort was 57 . There was a statistically higher ESR in the group of patients with a positive biopsy compared to the group with a negative biopsy. This supports the use of ESR in the initial diagnosis of GCA.

CRP is another biochemical marker that has frequently been shown to be raised in GCA. Our cohort showed no significant difference between mean CRP in those with a positive and those with a negative biopsy. This would support the use of ESR rather than CRP in the ACR diagnostic criteria for GCA. This analysis was limited by the lower than expected numbers of patients having recorded CRP prior to biopsy. BMI was also recorded on a small cohort of our patients. There was a significantly lower mean BMI in patients with positive biopsies than those with negative. This same phenomenon has previously been illustrated in other studies, ${ }^{15,16}$ and suggests that low BMI could be another useful predictive marker for patients with suspected GCA. The pathophysiology behind this is uncertain; however, it has been suggested that this may in part be due to the effect of reduced adipose tissue on hormonal pathways regulating inflammation. ${ }^{16}$

\section{Conclusion}

A large number of TABs are performed by several different specialities in the south-east of Scotland each year. This study shows that a large number of these biopsies are negative, and finding ways to reduce the number of patients unnecessarily undergoing biopsy will be essential in reducing workload and streamlining services. This study demonstrates some key predictive factors for patients with positive biopsies, including age, raised ESR and low BMI. The study also shows that a large proportion of biopsies taking place do not result in the recommended length of specimen, but this does not necessarily reduce the likelihood of a positive biopsy. (1)

\section{Appendix 1}

A correlated component regression has been developed to regularise regression for linear and logistic modelling as a means of reducing prediction error. In this case, regularisation is achieved through component/dimension reduction strategies as shown by Magidson. ${ }^{17}$ The purpose is to optimise $\mathrm{R}$ squared by manipulating both the number of correlated components $(k)$ and the predictors $(p)$ in any model. ${ }^{17}$ The correlated component regression also replaces $\mathrm{p}$ values by cross validated out-of-sample performance as provided in the predictor table (see below).

The dependent variable for the regression logistic regression is biopsy result. Predictors entered into the model were age at biopsy, history of fever/sweats, symptoms of polymyalgia, jaw claudication, headache, ophthalmic symptoms, fatigue, temporal tenderness, erythrocyte sedimentation rate, C-reactive protein, diameter of biopsy, gender, biopsy length, days from presentation to biopsy, scalp tenderness, referring specialty, grade of surgeon performing biopsy and TAB performing specialty.

Output from the regression showed that the cross validated solution was optimised with six predictors where $\mathrm{R}$ squared $=$ 0.108 and area under the curve $=0.687$. The following table lists the detail of the six predictor solution. As a measure of the importance or contribution of each predictor to the model, Pratt's coefficient is used. ${ }^{18}$ 
S Gajree, S Borooah, N Dhillon et al.

Table 1 Correlated component regression model of predictors of biopsy outcome

$\begin{array}{lll}\text { Model Fit Training Cross validation } & \text { C }\end{array}$

\begin{tabular}{lll}
\hline R squared & 0.156 & $0.108(\mathrm{se}=0.006)$ \\
\hline Area under the curve & 0.722 & $0.687(\mathrm{se}=0.006)$ \\
\hline Accuracy & 0.708 & $0.683(\mathrm{se}=0.005)$ \\
\hline
\end{tabular}

Predictors

Standard coefficient

Predictor Table

Freq $-\max \mathbf{n}=\mathbf{5 0 0}$

Pratt's coefficient of importance \%

\begin{tabular}{llll}
\hline Age at biopsy & 0.723 & 500 & $19 \%$ \\
\hline Specialty performing TAB & 0.705 & 500 & $18 \%$ \\
\hline Jaw claudication & 0.818 & 500 & $19 \%$ \\
\hline Ophthalmic symptoms & 0.525 & 500 & $10 \%$ \\
\hline Jaw pain & 0.687 & 500 & $13 \%$ \\
\hline Erythrocyte sedimentation rate & 0.734 & 500 & $21 \%$ \\
\hline
\end{tabular}

Next in rank but not in model

Diameter

0.217

468

\section{References}

1 Jennette JC, Falk RJ, Bacon PA et al. 2012 revised International Chapel Hill Consensus Conference Nomenclature of Vasculitides. Arthritis Rheum 2013; 65: 1-11.

2 Borchers AT, Gershwin ME. Giant cell arteritis: A review of classification, pathophysiology, geoepidemiology and treatment. Autoimmun Rev 2012; 11: A544-54.

3 Salvarani C, Cantini F, Boiardi L et al. Polymyalgia rheumatica and giant-cell arteritis. N Engl J Med 2002; 347: 261-71.

4 Aiello PD, Trautmann JC, McPhee TJ et al. Visual prognosis in giant cell arteritis. Ophthalmology 1993; 100: 550-5.

5 Hunder GG, Bloch DA, Michel BA et al. The American College of Rheumatology 1990 criteria for the classification of giant cell arteritis. Arthritis Rheum 1990; 33: 1122-8.

6 Dasgupta B, Borg FA, Hassan N et al. BSR and BHPR guidelines for the management of giant cell arteritis. Rheumatology 2010; 49: 1594-7.

7 Nesher G. The diagnosis and classification of giant cell arteritis. J Autoimmun 2014; 48-49: 73-5.

8 Le K, Bools LM, Lynn AB et al. The effect of temporal artery biopsy on the treatment of temporal arteritis. Am J Surg 2015; 209: 338-41.

9 Mukhtyar C, Guillevin L, Cid MC et al. EULAR recommendations for the management of large vessel vasculitis. Ann Rheum Dis 2009; 68: 318-23.

10 Achkar AA, Lie JT, Hunder GG et al. How does previous corticosteroid treatment affect the biopsy findings in giant cell (temporal) arteritis? Ann Intern Med 1994; 120: 987-92.
11 Moutray TN, Williams MA, Best JL. Suspected giant cell arteritis: a study of referrals for temporal artery biopsy. Can J Ophthalmol 2008; 43: 445-8.

12 Ypsilantis E. Courtney ED, Chopra N et al. Importance of specimen length during temporal artery biopsy. Br J Surg 2011; 98: 1556-60.

13 McMurran AE, Boom SJ. Temporal artery biopsies: do they make the cut? Scott Med J 2015 60: 9-12.

14 Mahr A, Saba M, Kambouchner M, et al. Temporal artery biopsy for diagnosing giant cell arteritis: the longer, the better? Ann Rheum Dis 2006; 65: 826-8.

15 Larsson K, Mellström D, Nordborg E et al. Early menopause, low body mass index, and smoking are independent risk factors for developing giant cell arteritis. Ann Rheum Dis 2006; 65: 529-32.

16 Ungprasert P, Thongprayoon C, Warrington KJ. Lower body mass index is associated with a higher risk of giant cell arteritis: a systematic review and meta-analysis. Ann Trans/ Med 2015; 3: 232.

17 Magidson J. Correlated component regression: Re-thinking regression in the presence of near collinearity. In: Abdi H, Chin WW, Esposito Vinzi V, et al., editors. New Perspectives in Partial Least Squares and Related Methods. New York: Springer Verlag; 2013. p.65-78.

18 Nathans LL,Oswald FL, Nimon K. Interpreting multiple linear regression: a guidebook of variable importance. Practical Assessment, Research and Evaluation 2012; 17 (9). http:// pareonline.net/getvn.asp? $v=17 \& n=9$ (accessed 25/5/17). 